Revista Científica do Corpo de Bombeiros Militar de Pernambuco

XVIII Seminário Nacional de Bombeiros - Foz do Iguaçu PR

Vol.04 No11 - Edição Especial XVIII SENABOM - ISSN 2359-4829

Versão on-line disponível em: http://www.revistaflammae.com.

\title{
RISCO CARDIOMETABÓLICO DA ATIVIDADE DE BOMBEIRO: ESTRATÉGIAS INDIVIDUAIS E INSTITUCIONAIS NA REDUÇÃO DO RISCO E NA PROMOÇÃO DA SAÚdE, COM DESTAQUE PARA O PAPEL DA APTID ÃO FÍSICA
}

\author{
CARDIOMETABOLIC RISK OF FIREFIGHTERS' DUTIES: INDIVIDUAL AND \\ INSTITUTIONAL STRATEGIES TO RISK REDUCTION AND HEALTH PROMOTION, \\ HIGHLIGHTING THE ROLE OF PHYSICAL FITNESS
}

Luiz Guilherme Grossi Porto ${ }^{1}$

\section{Resumo}

Trata-se de uma revisão não-sistemática sobre os principais riscos cardiometabólicos associados às atividades laborais de bombeiros. São abordadas estratégias individuais e institucionais com vistas à redução dos riscos inerentes à profissão de bombeiro e à promoção da saúde, com destaque para o papel da aptidão física. São inicialmente apresentadas as evidências científicas da relação entre atividade física e saúde. Sequencialmente, aborda-se a epidemiologia do risco cardiometabólico da atividade de bombeiro e o papel da aptidão física; a identificação de condições que tornam alguns indivíduos mais suscetíveis a eventos cardíacos e os desafios da triagem médicoocupacional. Como conclusão, são apresentadas sugestões práticas e recomendações com vistas à redução do risco cardiometabólico e à promoção da saúde de bombeiros.

Palavras-chave: Aptidão física. Risco cardiovascular. Cardiomegalia. Treinamento físico. Saúde.

\begin{abstract}
We conducted a non-systematic review about the main cardiometabolic risks associated to firefighters' duties. Individual and institutional strategies were addressed toward firefighters' duty-related risk reduction and health promotion, highlighting the role of physical fitness. Firstly, we presented the scientific evidences on the relationship between physical activity and health. Afterwards, we presented the epidemiology of the cardiometabolic risk of firefighting and the role of physical fitness; the identification of the conditions that contribute for greater susceptibility of some individuals for cardiovascular events and the challenge of the occupational medical screening. As conclusion, we presented some practical suggestions and recommendations toward the cardiometabolic risk reduction and firefighters' health promotion.
\end{abstract}

Key words: Physical fitness. Cardiovascular risk. Cardiomegaly. Physical training. Health.

1: Professor da Faculdade de Educação Física da Universidade de Brasília - UnB. Graduado em Educação Física, Mestre em Ciências da Saúde, Doutorado pela Faculdade de Medicina - UnB; Pós-doutorado na Harvard T.H. Chan School of Public Health - luizggporto@gmail.com 
Revista Científica do Corpo de Bombeiros Militar de Pernambuco

XVIII Seminário Nacional de Bombeiros - Foz do Iguaçu PR

Vol.04 No11 - Edição Especial XVIII SENABOM - ISSN 2359-4829

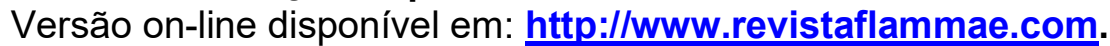

1 - A relação entre a atividade física e a saúde: visão histórica e recomendações gerais

Nas últimas décadas a relação entre atividade física e saúde se tornou tema frequente nas publicações leigas e científicas. No contexto científico, um bom indicador deste fato é o número de artigos publicados sobre o assunto nos últimos anos. Em consulta realizada em outubro de 2018 a uma das maiores bases mundiais de artigos científicos na área da saúde (Medline) por artigos com os termos saúde e atividade física (ATF) ou exercício no título, foram encontrados 4656 artigos e uma clara tendência de crescimento dessas publicações ao longo dos últimos 25 a 30 anos.

Historicamente, o estudo da relação entre o exercício ou o nível de atividade física (NATF) e o estado de saúde se concentrou na avaliação dos efeitos do treinamento físico sistemático sobre variáveis funcionais de desempenho, como o consumo máximo de oxigênio $\left(\mathrm{VO}_{2} \mathrm{máx}\right)$. Pesquisa pioneira conduzida por Morris e colaboradores, publicada em 1953, é considerada um marco histórico na área. Os autores avaliaram de forma sistemática a relação entre a ATF naturalmente desenvolvida no ambiente do trabalho e seus benefícios cardiovasculares. Este estudo evidenciou um aumento do risco de doença arterial coronariana entre os motoristas dos famosos ônibus de dois andares da cidade de Londres, na Inglaterra, comparativamente aos trabalhadores responsáveis pela cobrança da passagem, que desenvolviam sua atividade laboral em constante movimento entre os dois andares do veículo (MORRIS et al., 1953). Desde então, houve grande desenvolvimento dos estudos de cunho epidemiológico, com abordagem da relação atividade física-saúde e/ou exercício-saúde em populações diversas.

Nesse contexto, é importante considerar a distinção conceitual entre os termos "atividade física", "exercício" e "esporte". A ATF é definida como todo movimento humano executado pela musculatura esquelética que proporciona gasto energético superior ao de repouso. Já o exercício físico é uma subcategoria de ATF, que se caracteriza por ser planejada e estruturada, com objetivo de desenvolver a aptidão física. Esta, por sua vez, pode ser subdividida em componentes que visam à promoção da 
Revista Científica do Corpo de Bombeiros Militar de Pernambuco

XVIII Seminário Nacional de Bombeiros - Foz do Iguaçu PR

Vol.04 N011 - Edição Especial XVIII SENABOM - ISSN 2359-4829

Versão on-line disponível em: http://www.revistaflammae.com.

saúde ou ao aprimoramento do desempenho físico. Os componentes da aptidão física ligada à saúde são: capacidade aeróbica, força muscular, resistência muscular, flexibilidade e composição corporal. Já o esporte se diferencia das outras duas modalidades de prática física por envolver competiçaões, sujeitas a regras específicicas, formais ou não (CASPERSEN et al., 1985). No ambiente laboral, um bom exemplo é a atividade executada para a conferência de equipamentos dentro das unidades operacionais, que envolvam deslocamentos e/ou transporte de materiais, que se caracteriza como ATF, mas não é um exercício formal.

Estudos clássicos evidenciaram benefícios da maior aptidão física e/ou do NATF em vários desfechos em saúde, como na saúde cardiovascular (SESSO et al., 2000), na saúde metabólica (KNOWLER et al., 2002) e na longevidade (BLAIR et al., 1989; LEE e PAFFENBARGER, 2000). As evidências atuais dos vários benefícios para a saúde associados à prática regular de ATF e/ou à boa aptidão física são inegáveis. (LEE et al., 2012; EKELUND et al., 2016; SAINT-MAURICE et al., 2018). Entretanto, dados recentemente publicados indicam que praticamente um terço das mulheres e um quarto dos homens da população mundial ainda são insuficientemente ativos. (GUTHOLD et al., 2018). A insuficiência de ATF se configura hoje como um dos principais fatores de risco para mortalidade global, associando-se a mais de cinco milhões de mortes por ano. (LEE et al., 2012). Em contrapartida, suficiente nível de ATF, pouco tempo sentado e/ou a boa aptidão física se associam a inúmeros benefícios para a saúde, como menor mortalidade cardiovascular e global (MYERS et al., 2002; KATZMARZYK et al., 2009; OWEN et al., 2009), menor risco para hipertensão arterial (Al-MALLAH et al., 2018), diabetes melitus (KNOWLER et al., 2002), depressão, obesidade e alguns típos de canceres, especialmente de cólon e de mama (AREM et al., 2015; REZENDE et al., 2018; U.S. DEPARTMENT OF HEALTH AND HUMAN SERVICES, 2018).

Frente à crescente produção dessas evidências científicas, em 1995 o American College of Sports Medicine - ACSM, em conjunto com o Center for Disease Control and Prevention dos Estados Unidos $(C D C)$ publicaram a primeira recomendação formal de ATF para a saúde pública, preconizando que todo adulto deveria acumular pelo 


\section{Revista FLAMMAE}

Revista Científica do Corpo de Bombeiros Militar de Pernambuco

XVIII Seminário Nacional de Bombeiros - Foz do Iguaçu PR

Vol.04 No11 - Edição Especial XVIII SENABOM - ISSN 2359-4829

Versão on-line disponível em: http://www.revistaflammae.com.

menos 30 minutos de ATF moderada na maioria dos dias da semana e preferencialmente todos os dias (PATE et al., 1995). Esta recomendação foi atualizada em 2007 incorporando uma frequência mínima de 5 dias de ATF moderada por semana (HASKELL et al., 2007). Em 2010, a Organização Mundial da Saúde - OMS publicou sua recomendação própria, também preconizando o mínimo de 150 minutos de ATF moderada por semana, entretanto sem estabelecer a frequência semana mínima na qual esses 150 minutos poderiam ser acumulados (WORLD HEALTH ORGANIZATION., 2010). Todas essas recomendações também preveem a possibilidade de cumprimento do mínimo de ATF semanal com atividades vigorosas $(60 \mathrm{~min} / \mathrm{sem}$ em no mínimo 3 dias, pelo ACSM ou $75 \mathrm{~min} / \mathrm{sem}$ pela OMS), ou qualquer combinação equivalente de ATF moderadas e vigorosas.

\section{2 - A epidemiologia do risco cardiometabólico da atividade de bombeiro e o papel} da aptidão física

\section{1 - Risco cardiometabólico da atividade de bombeiro: fatores determinantes e suscetibilidade}

Inicialmente é importante enfatizar que bombeiros, assim como qualquer outra pessoa, devem ter como meta o cumprimento do mínimo de ATF recomenda para saúde. Entretanto, há que se ponderar se o profissional bombeiro deveria atingir patamares superiores de ATF e/ou de aptidão física, comparativamente à população em geral. Ademais, é também importante avaliar se uma aptidão física elevada representa um fator protetor para a saúde do bombeiro, além de seu potencial benefício para o desempenho profissional.

Sabe-se que a atividade de bombeiro envolve elevadas demandas físicas e emocionais. Muitas tarefas inerentes ao seu desempenho profissional impõem elevada sobrecarga cardiovascular, podendo resultar em situações críticas como a morte súbita cardíaca e doenças cardiovasculares precoces, afetando tanto a saúde quanto a qualidade 
de vida e a capacidade laboral desses profissionais (SOTERIADES et al., 2011; KALES e SMITH, 2017; GAINEY et al., 2018).

As atividades de busca e salvamento, de combate a incêndios urbano ou florestal e/ou de atendimento de emergências médicas são algumas das ações que caracterizam a rotina de trabalho de bombeiros. Somam-se ainda outros fatores como o trabalho em regime de escala, jornadas de trabalho extraordinárias e o baixo poder decisório nas ações, entre outros. A literatura especializada abordando os riscos à saúde inerentes à profissão de bombeiro vem crescendo, tendo em vista as evidências que indicam que esses profissionais são sujeitos a riscos à saúde muito superiores àqueles que afetam a população em geral. Destacam-se neste cenário os riscos cardiovasculares, metabólicos e de desenvolvimento de tumores malignos e de distúrbios psicológicos graves (KALES et al., 2009; KALES e SMITH, 2017; GAINEY et al., 2018).

Um estudo balizador na área, publicado em 2007, indicou que 45\% das mortes de bombeiros americanos em serviço eram causadas por doenças cardíacas. Analisando a associação das causas de morte com o tipo de atividade profissional desenvolvida, os autores observaram que $32,1 \%$ das mortes ocorreram no combate a incêndios, $13,4 \%$ durante o atendimento ao alarme (chamado), 17,4\% no retorno após atendimento, 12,5\% em situações de treinamento físico, 9,5\% em atendimentos a emergências sem fogo e 15,4\% em situações variadas de não-emergência. (KALES et al., 2007). Destacase que apesar dos bombeiros do estudo passarem somente entre 1 a $5 \%$ de seu tempo de trabalho em efetivo combate a incêndio, praticamente um terço das mortes em serviço ocorreram nesta condição. Assim, a atividade de combate a incêndio apresentou um risco de morte por doença arterial coronariana 136 vezes maior que aquele enfrentado em atividades de não-emergência. Do ponto de vista prático, esses achados sugerem fortemente que as análises de risco cardiovascular entre bombeiros devem considerar não só a condição individual, mas também o tipo de atividade a ser desempenhada.

Tendo por base a premissa epidemiológica de que eventos em saúde pública não ocorrem ao acaso, o conhecimento dos possíveis fatores determinantes é fundamental para a adoção de medidas de prevenção de riscos e de promoção da saúde. Como se 
observa no estudo de Kales e colaboradores de 2007, as mortes em serviço de origem cardiovascular não são aleatórias quanto ao tipo de atividade desenvolvida, sendo o combate a incêndio a atividade de maior risco (KALES et al., 2007). Smith e colaboradores evidenciaram nesse cenário que cerca de 45 a $50 \%$ das mortes em serviço de bombeiros americanos entre os anos de 1990 a 2014 foram de origem cardíaca, caracterizando-se como a principal causa de morte em serviço nesses profissionais, contrariando o senso comum, que indicaria os casos de asfixia, de queimaduras ou de colapso de estruturas como fator principal (SMITH et al., 2016). Além da atividade executada, os riscos à saúde de bombeiros também não se mostram aleatórios quanto à distribuição dos eventos ao longo das estações do ano e/ou nas diferentes horas do dia (SOTERIADES et al., 2011). Na esfera da saúde ocupacional é fundamental identificar os principais riscos inerentes à profissão a que os bombeiros são submetidos, assim como quais fatores podem ter papel protetor. Sabe-se que a maioria dos bombeiros se recuperará do estresse decorrente do combate a incêndio e/ou de outras atividades profissionais. Entretanto, a sobrecarga cardiovascular inerente a algumas ações profissionais, com destaque para o combate a incêndio, pode representar um gatilho para a ocorrência de eventos cardíacos graves em indivíduos suscetíveis (SMITH et al., 2016). O desafio, portanto, recai sobre a identificação precoce de indivíduos mais suscetíveis, com vistas à adoção de ações de tratamento da saúde, de prevenção de aumento de riscos e de promoção da saúde.

Digno de nota o fato de que boa parte dos fatores agressores à saúde de bombeiros já é conhecida, podendo-se destacar a exposição à fumaça, ao ruído, o trabalho em regime de escala, a privação de sono, a existência de elevadas demanda físicas e emocionais específicas da atividade, o baixo nível de controle decisório, o treinamento físico irregular, entre outros (SOTERIADES et al., 2011). Neste cenário há que se considerar ainda que condições como a baixa aptidão física, a obesidade e a hipertensão arterial, que sabidamente aumentam o risco cardiovascular em situações de elevada demanda física e/ou emocional, também podem estar presentes entre esses profissionais, agravando os riscos (SOTERIADES et al., 2005; KALES et al., 2009; 
YANG et al., 2013; NOGUEIRA et al., 2016; KORRE et al., 2018; SEGEDI e PORTO, 2018).

Neste cenário, Kales \& Smith propuseram em 2017 um modelo teórico de descrição da sobrecarga cardiovascular das atividades de combate a incêndio em associação com características individuais dos bombeiros. Esse modelo agrega as condições de risco inerentes à profissão e aquelas relativas a características pessoas que podem aumentar ou reduzir o risco do desencadeamento de eventos cardíacos súbitos que, por sua vez, podem levar à morte subida cardíaca em serviço (KALES e SMITH, 2017). Os autores apresentam didaticamente quatro grandes conjuntos de fatores que podem, isoladamente ou de forma conjunta, funcionar como gatilho para a ocorrência eventos cardiovasculares agudos de grande magnitude. No conjunto dos estressores fisiológicos associados à atividade laboral, incluem-se: a) os ajustes autonômicos do coração com destaque para a ativação do sistema nervoso simpático frente ao atendimento próximo, desde o acionamento do alarme (brado) até o enfrentamento do evento; b) o esforço físico propriamente dito, muita vezes acrescido de pesados equipamentos de proteção individual; c) o estresse térmico e a desidratação e; d) as condições ambientais, como a presença de partículas poluentes no ar, a exposição à fumaça e/ou a substancias asfixiantes. $\mathrm{O}$ segundo conjunto diz respeito às características das atividades, nas quais os riscos são crescentes desde aquelas classificadas como de não-emergência, passando pelas emergências médicas pré-hospitalares, a resposta ao brado, o treinamento físico, o retorno do atendimento, até chegar àquela de maior risco, que é o combate ao incêndio. No terceiro conjunto de fatores são incluídas as respostas fisiológicas, que são afetadas pelas condições do bombeiro no período pré-plantão, como nível de repouso e de hidratação prévios. Os riscos aumentam com o aumento do estresse de cisalhamento vascular, com a redução do volume plasmático, com alteração de eletrólitos, do aumento da viscosidade do sangue e de um estado pró-coagulante. O último bloco do modelo refere-se a características individuais, como idade, sexo, estilo de vida, estado de saúde e aptidão física. Boas condições de saúde e de aptidão física reduzem os riscos, ao passo que o excesso de peso, a baixa aptidão física, a presença de 
fatores de risco para doenças cardiovasculares e a obesidade representam aumento de risco, que poderá ser ainda maior quando da presença de doenças clínicas ou subclínicas e na presença de doença coronariana associada ou não à cardiomegalia. Os autores propõem então que existe um limiar individual de sobrecarga que, se ultrapassado, pode desencadear eventos cardíacos súbitos graves, fatais ou não-fatais. Na maioria dos casos esse liminar não é ultrapassado e os bombeiros passaram por um período de recuperação para a condição de equilíbrio basal (homeostase) (KALES e SMITH, 2017). Deve-se almejar então a redução dos fatores que aumentam os riscos e, portanto, a chance de que o limiar individual seja ultrapassado. Considerando que existem exigências de elevadas intensidades que são inerentes à profissão e, por conseguinte, não são evitáveis (como o combate a incêndios), o enorme desafio no âmbito da saúde ocupacional é na identificação precoce de indivíduos mais suscetíveis, ou seja, aqueles que por condições individuais (temporárias ou permanentes), associadas às características das tarefas a serem desenvolvidas, tenham mais chance de que o limiar individual de sobrecarga seja ultrapassado.

\section{2 - Redução do risco cardiometabólico: um enfoque na aptidão física para a saúde}

A partir do conhecimento de vários fatores determinantes, torna-se possível a análise de estratégias para redução de riscos e a promoção da saúde. Uma estratégia fundamental a ser considerada é a redução dos fatores de risco clássicos para doenças cardiometabólicas, como obesidade, tabagismo e hipertensão, entre outros. Entretanto, uma importante ressalva se faz necessária quando se avalia a presença de fatores de risco para doenças crônico não-transmissíveis, com destaque para as cardiometabólicas, entre categorias profissionais específicas, como no caso dos bombeiros. Ainda que a profissão se caracterize por elevadas demandas físicas e/ou emocionais, para as quais se preconiza de modo geral um "elevado padrão de saúde", com boa aptidão física, adequada composição corporal e ausência de fatores de risco cardiovasculares, entre outros, é fundamental considerar que esses profissionais estão inseridos nas suas respectivas sociedades e, portanto, também sujeitos a fatores de exposição e 
Revista Científica do Corpo de Bombeiros Militar de Pernambuco

XVIII Seminário Nacional de Bombeiros - Foz do Iguaçu PR

Vol.04 No11 - Edição Especial XVIII SENABOM - ISSN 2359-4829

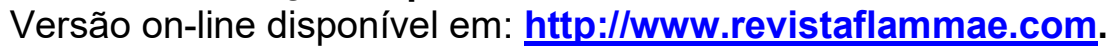

determinantes sociais da saúde semelhantes aos da população em geral, como fatores ambientais e comportamentais da sociedade em que se vive (LAKERVELD e MACKENBACH, 2017; KORRE et al., 2018). Um bom exemplo deste fato é um estudo publicado em 2011 que identificou prevalência de obesidade entre bombeiros americanos similar àquela observada na população em geral (POSTON et al., 2011). No cenário brasileiro, estudo recentemente concluído em nosso grupo apontou um quadro de menor prevalência de obesidade entre bombeiros, no ano de 2017, comparativamente ao observado da população geral de origem, o que foi diferente de achado anterior, relativo ao ano de 2009, onde a prevalência de obesidade era similar à da população em geral. (NOGUEIRA et al., 2016; SEGEDI, 2018). Apesar de diferenças metodológicas que limitam a comparação desses resultados, é digno de nota que a menor prevalência de obesidade identificada em 2017 comparativamente a 2009 coincide com a inauguração de um moderno centro de capacitação física e a implantação de políticas internas, como a criação de academias de ginástica nas unidades operacionais e a valorização do treinamento físico. Os dados são promissores e merecem uma avaliação longitudinal que possa avaliar se existe, ou não, efetiva relação de associação entre esses fatores.

Outra questão a ser considerada são os ajustes cardiovasculares agudos associados ao esforço físico, frente às diferentes demandas a que os trabalhadores da segurança pública estão expostos no exercício de suas funções e sua relação com os riscos da profissão. A literatura publicada nos últimos 40 anos é vasta em relação às análises das respostas agudas do aparelho cardiovascular frente ao exercício físico, em diferentes modalidades e intensidades. No particular, estudos tem apontado associação clara entre a aptidão física e a composição corporal e os riscos à saúde de bombeiros e agentes de segurança (BAUR et al., 2012; MILESKI et al., 2015). Importante mencionar nesse cenário que dados do nosso grupo confirma outros achados que demonstram a ocorrência de picos, de duração variada, de elevada demanda cardiovascular no exercício profissional, tanto urbano quanto florestal (NOGUEIRA e PORTO, 2016; SAINT-MARTIN e PORTO, 2018). 
Revista Científica do Corpo de Bombeiros Militar de Pernambuco

XVIII Seminário Nacional de Bombeiros - Foz do Iguaçu PR

Vol.04 N011 - Edição Especial XVIII SENABOM - ISSN 2359-4829

Versão on-line disponível em: http://www.revistaflammae.com.

O papel protetor conferido por uma melhor aptidão cardiorrespiratória (ACR) tem sido evidenciado em diferentes desfechos entre profissionais bombeiros. Baur e colaboradores identificaram uma clara, significativa e inversa associação entre a ACR e a síndrome metabólica (SM), que é uma condição que aumenta o risco cardiovascular. Este estudo avaliou 957 bombeiros americanos com média de idade de 39,6 anos e identificou uma prevalência de $28,3 \%$ de SM. Destaca-se que a prevalência de SM foi 10 vezes maior no grupo com menor ACR $(</=10$ METs) (BAUR, CHRISTOPHI, et al., 2012). Um outro estudo do mesmo grupo também identificou associação significativa e inversa entre a ACR e a presença de anormalidades eletrocardiográficas e autonômicas durante o teste de esforço cardiológico em bombeiros assintomáticos. Os autores concluíram que bombeiros com ACR $<12$ METS devem receber atenção para a redução do risco cardiovascular, incluindo a melhora da ACR.

Estudo conduzido entre bombeiros brasileiros identificou clara associação entre a ACR e a composição corporal. Em amostra de 4.237 bombeiros do sexo masculino, observamos que aqueles com indicadores de composição corporal dentro da normalidade (avaliada por 3 métodos) apresentaram ACR aproximadamente 1,3 MET acima daqueles com composição corporal acima dos valores de normalidade. É importante destacar que a NFPA (United States National Fire Protection Association) preconiza que todo bombeiro deve ter ACR mínima de 12 METS para o desempenho seguro de suas tarefas (NFPA, 2007). No estudo acima mencionado, observamos que a possibilidade do bombeiro não atingir a recomendação de um mínimo de 12 METs era entre 2,9 e 8,1 vezes maior entre aqueles com composição corporal acima do recomendado, comparativamente àqueles com adequada composição (NOGUEIRA et al., 2016). Destaca-se que a adequada ACR, além de preconizada pela NFPA, é variável que consta em todos os modelos teóricos de análise de riscos da atividade de bombeiros (SOTERIADES et al., 2011; SMITH et al., 2016; KALES e SMITH, 2017). 
Revista Científica do Corpo de Bombeiros Militar de Pernambuco

XVIII Seminário Nacional de Bombeiros - Foz do Iguaçu PR

Vol.04 No11 - Edição Especial XVIII SENABOM - ISSN 2359-4829

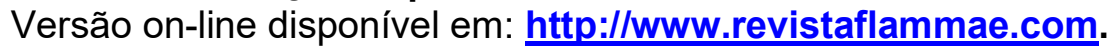

3 - Susceptibilidade e condições clínicas predisponentes: desafios da triagem médico-ocupacional

Conforme comentado, as condições de sobrecarga cardiometabólica agudas são bem toleradas pela maioria dos bombeiros. Entretanto, algumas condições clínicas e/ou subclínicas, associadas ou não a outras características pessoais e/ou com características da tarefa a ser executada, colocam alguns indivíduos em situação de maior susceptibilidade. Considerando que do ponto de vista da tarefa está bem documentado que o combate ao incêndio é aquela que representa maior risco (KALES et al., 2007; FARIOLI et al., 2014; KALES e SMITH, 2017), o desafio recai sobre a avaliação de características dos indivíduos, que associadas às atividades extenuantes como o combate ao incêndio, podem caracterizar-se como condição de grande aumento de risco. Algumas possibilidades de mecanismos fisiopatológicos associados à morte súbita cardíaca têm sido propostos, a exemplo do aumento do trabalho do coração, da disfunção vascular, da isquemia tecidual e de um estado pró-coagulante (Smith et al., 2016). Em paralelo, estudo publicado há 10 anos identificou que a existência de doença coronariana prévia, o fumo e a hipertensão se mostraram como fortes preditores de eventos fatais em serviço entre bombeiros (GEIBE et al., 2008). Os autores sugerem que a prevenção deve incluir a deteç̧ão e controle da hipertensão arterial, apoio para a suspensão do tabagismo e a possibilidade de restrições laborais para bombeiros com significativa doença arterial coronariana. Em bombeiros jovens $(</=45$ anos), resultados semelhantes foram identificados, no sentido em que a maior parte dos preditores para morte subida cardíaca estava relacionada a condições muitas vezes preveníeis, como a obesidade, a hipertensão arterial e o tabagismo. (YANG et al., 2013). Ademais, também em bombeiros mais novos, o histórico de condição cardíaca prévia aumentou significativamente o risco de morte súbita (FARIOLI et al., 2014).

Quanto às condições clínicas ou sub-clínicas, a literatura tem evidenciado tanto alterações estruturais quanto funcionais do aparelho cardiovascular associadas ao aumento de risco. Dentre as alterações estruturais, a presença de cardiomegalia e/ou de hipertrofia ventricular esquerda, além da doença coronariana prévia, aparecem como 
preditores da morte súbita cardíaca (YANG et al., 2013; SMITH et al., 2016; KALES e SMITH, 2017; SMITH et al., 2018). Do ponto de vista funcional, a baixa aptidão física e alterações na regulação autonômica do coração são questões a serem consideradas. A Figura 1 ilustra interações possíveis dessas condições, que associadas às características individuais e da tarefa podem representar aumento de risco de eventos cardiovasculares súbitos.

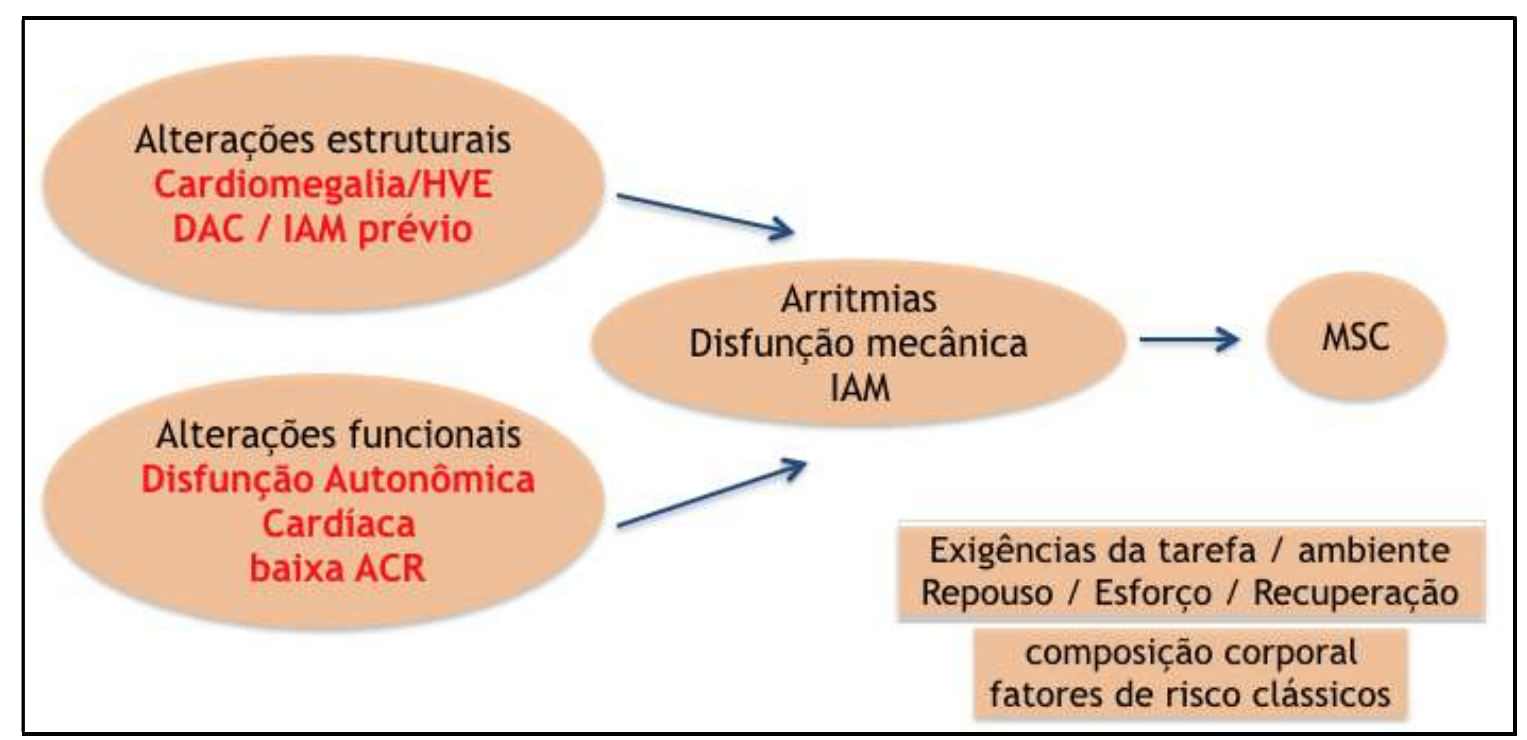

Figura 1: Modelo simplificado de interações estruturais e funcionais que, em associação com características individuais e da tarefa a ser executada, podem resultar em eventos cardiovasculares súbitos durante atividades laborais de bombeiros. Legenda: HVE: hipertrofia ventricular esquerda; DAC: doença arterial coronariana; IAM: infarto agudo do miocárdio; ACR: aptidão cardiorrespiratória; MSC: morte súbita cardíaca.

Estudo muito recentemente publicado, baseado em dados de autópsias de bombeiros norte-americanos falecidos em serviço entre 1999 e 2014, evidenciou que $82 \%$ dos casos de morte de origem cardíaca estavam associadas à doença coronariana $\mathrm{e}$ à cardiomegalia, sendo essas duas condições independentemente associadas a elevação significativa do risco de morte súbita cardíaca. Frente a essas evidências, os autores sugerem que a inclusão de triagem para a doença arterial coronariana, para o aumento da massa do coração e para a existência prévia de infarto do miocárdio deve ser considerada, visando a prevenção de mortes cardíacas relacionadas ao trabalho entre bombeiros. 
Revista Científica do Corpo de Bombeiros Militar de Pernambuco

XVIII Seminário Nacional de Bombeiros - Foz do Iguaçu PR

Vol.04 No11 - Edição Especial XVIII SENABOM - ISSN 2359-4829

Versão on-line disponível em: http://www.revistaflammae.com.

\section{4 - Sugestões e recomendações práticas}

Com base nas evidências científicas disponíveis, apresentam-se abaixo algumas sugestões e recomendações práticas visando a redução do risco cardiometabólico e a promoção da saúde de bombeiros. Importante mencionar que os tópicos abaixo devem ser sempre avaliados por cada instituição quanto à sua viabilidade e adequação, pelos próprios profissionais e pelos responsáveis pela avaliação, treinamento e/ou tratamento do profissional bombeiro, quanto à adequação individual, possibilidades e/ou limitações, de acordo com potencialidades e condições clínicas de cada indivíduo. Tratam-se de sugestões / recomendações genéricas, que não necessariamente se aplicam a todos.

Desta forma, e visando a promoção da saúde e a redução do risco de doenças cardiometabólicas, as evidencias suportam, entre outras, as seguintes recomendações para os profissionais bombeiros sem restrições de saúde para as atividades operacionais:

1. Acumular pelo menos 150 min de ATF moderada (3 a 6 METS) por semana, ou 75 minutos de ATF vigorosa ( $\geq 6$ METS), ou qualquer combinação equivalente;

2. Instituir treinamento físico regular visando uma capacidade cardiorrespiratória $\geq 12$ METS, o que equivale a um $\mathrm{VO}_{2}=42,0$ $\mathrm{mL} \cdot \mathrm{kg}^{-1} \cdot \min ^{-1}$, e níveis adequados para a faixa etária e gênero das demais qualidades físicas da aptidão física para a saúde. O treinamento deve ser progressivo e respeitando as características individuais, uma vez que a realização de atividades vigorosas em não treinados pode representar elevado aumento de risco;

3. Reduzir o tempo sentado;

4. Instituir pausas ativas no tempo sentado;

5. Aumentar o acúmulo de atividades físicas leves no cotidiano;

6. Para além das atividades físicas, instituir estratégias para um sono suficiente e de qualidade, além de uma alimentação saudável. 
Para as recomendações acima, as evidências mais recentes apontam para a possibilidade de acúmulo de atividades físicas em qualquer duração, seguindo a lógica de que todo movimento conta.

Do ponto de vista das ações institucionais e de triagem no contexto da saúde ocupacional, respeitando-se características individuais, as evidências sugerem:

1. Instituir políticas públicas de estímulo à prática de ATF e do treinamento físico regular;

2. Instituir protocolos de avaliação física regular, a exemplo dos "Testes de Aptidão Física - TAFs", que poderão cumprir não só a função administrativa dos requisitos da carreira, mas também subsidiar avaliações em saúde;

3. Considerar a aptidão cardiorrespiratória e o NATF na avaliação global do risco cardiometabólico;

4. Considerar a inclusão de rotinas de rastreamento para doença arterial coronariana, cardiomegalia e/ou hipertrofia ventricular esquerda, além dos tradicionais fatores de risco cardiometabólico, como obesidade, hipertensão e diabetes, com vistas à detecção precoce de indivíduos mais suscetíveis à ocorrência de eventos cardiovasculares graves, e a consequente avaliação da adequação das tarefas laborais a serem desempenhadas.

Em conjunto, essas recomendações e sugestões, tanto no âmbito individual quanto no institucional, visam a redução do risco de agravos à saúde e a promoção da saúde e/ou do desempenho profissional de trabalhadores que enfrentam elevados riscos laborais, inerentes às características da profíssão, e que têm como missão última salvar vidas. Neste aspecto, toda e qualquer iniciativa desta natureza tende a aumentar as chances de sucesso desses profissionais, e instituições, nesta nobilíssima missão, assim como de preservar a integridade desses profissionais de elevadíssima relevância social.

\section{REFERÊNCIAS:}


Revista Científica do Corpo de Bombeiros Militar de Pernambuco

XVIII Seminário Nacional de Bombeiros - Foz do Iguaçu PR

Vol.04 N011 - Edição Especial XVIII SENABOM - ISSN 2359-4829

Versão on-line disponível em: http://www.revistaflammae.com.

AL-MALLAH, M. H.; SAKR, S.; AL-QUNAIBET, A. Cardiorespiratory Fitness and Cardiovascular Disease Prevention: an Update. Curr Atheroscler Rep, v. 20, n. 1, p. 1, Jan 162018.

AREM, $\mathrm{H}$. et al. Leisure time physical activity and mortality: a detailed pooled analysis of the dose-response relationship. JAMA Intern Med, v. 175, n. 6, p. 959-67, Jun 2015.

BAUR, D. M.; CHRISTOPHI, C. A.; KALES, S. N. Metabolic syndrome is inversely related to cardiorespiratory fitness in male career firefighters. J Strength Cond Res, v. 26, n. 9, p. 2331-7, Sep 2012.

BAUR, D. M. et al. Low fitness is associated with exercise abnormalities among asymptomatic firefighters. Occup Med (Lond), v. 62, n. 7, p. 566-9, Oct 2012.

BLAIR, S. N. et al. Physical fitness and all-cause mortality. A prospective study of healthy men and women. JAMA, v. 262, n. 17, p. 2395-401, Nov 31989.

CASPERSEN, C. J.; POWELL, K. E.; CHRISTENSON, G. M. Physical activity, exercise, and physical fitness: definitions and distinctions for health-related research. Public Health Rep, v. 100, n. 2, p. 126-31, Mar-Apr 1985.

EKELUND, U. et al. Does physical activity attenuate, or even eliminate, the detrimental association of sitting time with mortality? A harmonised meta-analysis of data from more than 1 million men and women. Lancet, v. 388, n. 10051, p. 1302-10, Sep 242016.

FARIOLI, A. et al. Duty-related risk of sudden cardiac death among young US firefighters. Occup Med (Lond), v. 64, n. 6, p. 428-35, Sep 2014.

GAINEY, S. J. et al. Exposure to a firefighting overhaul environment without respiratory protection increases immune dysregulation and lung disease risk. PLoS One, v. 13, n. 8, 2018.

GEIBE, J. R. et al. Predictors of on-duty coronary events in male firefighters in the United States. Am J Cardiol, v. 101, n. 5, p. 585-9, Mar 012008.

GUTHOLD, R. et al. Worldwide trends in insufficient physical activity from 2001 to 2016: a pooled analysis of 358 population-based surveys with 1.9 million participants. Lancet Glob Health, v. 6, n. 10, p. e1077-e1086, Oct 2018.

HASKELL, W. L. et al. Physical activity and public health: updated recommendation for adults from the American College of Sports Medicine and the American Heart Association. Med Sci Sports Exerc, v. 39, n. 8, p. 1423-34, Aug 2007.

KALES, S. N.; SMITH, D. L. Firefighting and the Heart: Implications for Prevention. Circulation, v. 135, n. 14, p. 1296-1299, Apr 2017. 
Revista Científica do Corpo de Bombeiros Militar de Pernambuco XVIII Seminário Nacional de Bombeiros - Foz do Iguaçu PR

Vol.04 No11 - Edição Especial XVIII SENABOM - ISSN 2359-4829

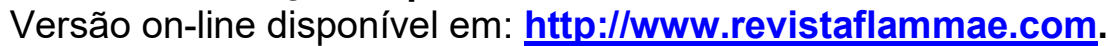

KALES, S. N. et al. Emergency duties and deaths from heart disease among firefighters in the United States. N Engl J Med, v. 356, n. 12, p. 1207-15, Mar 222007.

Blood pressure in firefighters, police officers, and other emergency responders. Am J Hypertens, v. 22, n. 1, p. 11-20, Jan 2009.

KATZMARZYK, P. T. et al. Sitting time and mortality from all causes, cardiovascular disease, and cancer. Med Sci Sports Exerc, v. 41, n. 5, p. 998-1005, May 2009.

KNOWLER, W. C. et al. Reduction in the incidence of type 2 diabetes with lifestyle intervention or metformin. N Engl J Med, v. 346, n. 6, p. 393-403, Feb 72002.

KORRE, M.; SMITH, D.; KALES, S. N. Obesity and health in the North American Fire Service: research points the way to positive culture change. Occup Med (Lond), v. 68, n. 3, p. 160-162, 052018.

LAKERVELD, J.; MACKENBACH, J. The Upstream Determinants of Adult Obesity. Obes Facts, v. 10, n. 3, p. 216-222, 2017.

LEE, I. M.; PAFFENBARGER, R. S., JR. Associations of light, moderate, and vigorous intensity physical activity with longevity. The Harvard Alumni Health Study. Am J Epidemiol, v. 151, n. 3, p. 293-9, Feb 12000.

LEE, I. M. et al. Effect of physical inactivity on major non-communicable diseases worldwide: an analysis of burden of disease and life expectancy. Lancet, v. 380, n. 9838, p. 219-29, Jul 212012.

MILESKI, K. S. L. et al. Health-related physical fitness in middle-aged men with and without metabolic syndrome. Journal of Sports Medicine and Physical Fitness, v. 55, n. 3, p. 223-230, MAR 20152015.

MORRIS, J. N. et al. Coronary heart-disease and physical activity of work. Lancet, v. 265, n. 6796, p. 1111-20; concl, Nov 1953.

MYERS, J. et al. Exercise capacity and mortality among men referred for exercise testing. The New England journal of medicine, v. 346, n. 11, p. 793-801, Mar 14 2002.

NFPA, N. F. P. A.-. NFPA 1582: Standard on Comprehensive Occupational Medical Program for Fire Departments. Quincy, MA. 2007

NOGUEIRA, E. C. et al. Body Composition is Strongly Associated With Cardiorespiratory Fitness in a Large Brazilian Military Firefighter Cohort: The Brazilian Firefighters Study. Journal of Strength and Conditioning Research, v. 30, n. 1, p. 33-38, 20162016.

NOGUEIRA, R.M. \& PORTO, L.G.G (orientador). Avaliação da função autonômica cardíaca e da sobrecarga cardiovascular de bombeiros militares durante turno de serviço 
Revista Científica do Corpo de Bombeiros Militar de Pernambuco

XVIII Seminário Nacional de Bombeiros - Foz do Iguaçu PR

Vol.04 No11 - Edição Especial XVIII SENABOM - ISSN 2359-4829

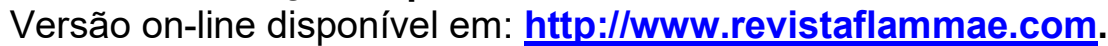

operacional. 2016. (Dissertação de Mestrado). Programa de Pós-Graduação em Educação Física, Universidade de Brasília

OWEN, N.; BAUMAN, A.; BROWN, W. Too much sitting: a novel and important predictor of chronic disease risk? Br J Sports Med, v. 43, n. 2, p. 81-3, Feb 2009.

PATE, R. R. et al. Physical activity and public health. A recommendation from the Centers for Disease Control and Prevention and the American College of Sports Medicine. Jama, v. 273, n. 5, p. 402-7, Feb 11995.

POSTON, W. S. et al. The prevalence of overweight, obesity, and substandard fitness in a population-based firefighter cohort. J Occup Environ Med, v. 53, n. 3, p. 266-73, Mar 2011.

REZENDE, L. F. M. et al. Physical activity and cancer: an umbrella review of the literature including 22 major anatomical sites and 770000 cancer cases. Br J Sports Med, v. 52, n. 13, p. 826-833, Jul 2018.

SAINT-MARTIN, D. R. \& PORTO, L.G.G (orientador). Avaliação objetiva do nível de atividade física, do comportamento sedentários e da aptidão cardiorrespiratória de bombeiros militares. 2018. (Dissertação de Mestrado). Programa de Pós-Graduação em Educação Física, Universidade de Brasília

SAINT-MAURICE, P. F. et al. Moderate-to-Vigorous Physical Activity and All-Cause Mortality: Do Bouts Matter? J Am Heart Assoc, v. 7, n. 6, Mar 2018.

SEGEDI, L \& PORTO, L.G.G (orientador). Análise da qualidade de vida, do nível de atividade física, da aptidão física e de fatores associados em bombeiros militares de ambos os gêneros. 2018. (Dissertação de Mestrado). Programa de Pós-Graduação em Educação Física, Universidade de Brasília

SESSO, H. D.; PAFFENBARGER, R. S., JR.; LEE, I. M. Physical activity and coronary heart disease in men: The Harvard Alumni Health Study. Circulation, v. 102, n. 9 , p. $975-80$, Aug 292000.

SMITH, D. L. et al. Cardiovascular Strain of Firefighting and the Risk of Sudden Cardiac Events. Exerc Sport Sci Rev, v. 44, n. 3, p. 90-7, Jul 2016.

SMITH, D. L et al. Pathoanatomic Findings Associated With Duty-Related Cardiac Death in US Firefighters: A Case - Control Study. J Am Heart Ass. 72018

SOTERIADES, E. S. et al. Obesity and cardiovascular disease risk factors in firefighters: a prospective cohort study. Obes Res, v. 13, n. 10, p. 1756-63, Oct 2005.

Cardiovascular Disease in US Firefighters A Systematic Review. Cardiology in Review, v. 19, n. 4, p. 202-215, Jul-Aug 2011. 
Revista Científica do Corpo de Bombeiros Militar de Pernambuco

XVIII Seminário Nacional de Bombeiros - Foz do Iguaçu PR

Vol.04 N011 - Edição Especial XVIII SENABOM - ISSN 2359-4829

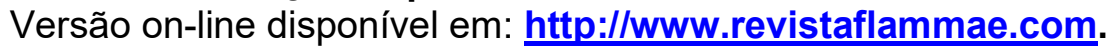

U.S. DEPARTMENT OF HEALTH AND HUMAN SERVICES. Physical Activity Guidelines Advisory Committee. Physical activity guidelines advisory committee scientific report 2018.

WORLD HEALTH ORGANIZATION. Global recommendations on physical activity for health. Geneva: World Health Organization: 58 p. p. 2010.

YANG, J. et al. Sudden cardiac death among firefighters $<=45$ years of age in the United States. Am J Cardiol, v. 112, n. 12, p. 1962-7, Dec 152013. 